Rajapaksha, M.R.N, Sridarran, P. and Rathnayake, R.M.D.I.M., 2021. Use of augmented reality for efficient building maintenance in Sri Lanka. In: Sandanayake, Y.G., Gunatilake, S. and Waidyasekara, K.G.A.S. (eds). Proceedings of the $9^{\text {th }}$ World Construction Symposium, 9-10 July 2021, Sri Lanka. [Online]. pp. 553-563. https://doi.org/10.31705/WCS.2021.48. Available from: https://ciobwcs.com/papers/

\title{
USE OF AUGMENTED REALITY FOR EFFICIENT BUILDING MAINTENANCE IN SRI LANKA
}

\author{
M.R.N Rajapaksha ${ }^{1}$, P. Sridarran ${ }^{2}$ and R.M.D.I.M. Rathnayake ${ }^{3}$
}

\begin{abstract}
With the advent of technological advancements in the field of building maintenance, attempts have been taken to address the issues confronted by building maintenance technicians in the global context. Augmented Reality (AR) is one such concept which combines real environment with virtual content in $3 D$ space that is experimented and proven to be effective in making the building maintenance activities more efficient by eliminating the causes for such difficulties faced by the technicians. In Sri Lanka, there is very limited use of such technologies in building maintenance practices and it is reasonable to mention that use of AR is minimum in this field. A gap is identified as there is no considerable literature exploring applications of Augmented Reality to ease building maintenance activities in Sri Lanka. Therefore, an effort is made to investigate into this area through a qualitative approach by taking a case into study and conducting interviews among technicians followed by an expert interview. The research highlights the use of $A R$ in making the building maintenance practices in terms of data and instruction visualization leading to more efficient and effective outcomes while discussing on the benefits such as making maintenance tasks efficient, cost and time savings and high customer satisfaction as well as possible barriers for implementation of a similar program such as developing a Building Information Model to an existing building, inconvenience in making technicians adopted into a new system in the context of Sri Lanka
\end{abstract}

Keywords: Applications of augmented reality; Augmented reality; Efficient building maintenance; Issues.

\section{INTRODUCTION}

Augmented reality technology has been adopted in numerous industries such as marketing, medicine, education, entertainment (Billinghurst et al., 2014), tourism, geometry modelling and scene construction, assembly and maintenance (Chatzopoulos et $a l ., 2017)$. In Sri Lanka, efforts of finding viability of AR application has been made areas such as education, Medicine, Urban planning and Marketing (Adikari et al., 2020; Chandike, 2016; Chandrasekara, 2015). According to Wang et al. (2013), Architecture/ design and construction is one prominent field that has caught considerable attention in research relating Augmented Reality and it is the idea of the researchers that integration of Augmented Reality with Facilities Management is still at an elementary level but it has great potential for evolvment. Especially in the Sri Lankan Context, there are no

\footnotetext{
${ }^{1}$ Department of Building Economics, University of Moratuwa, Sri Lanka, ravienarajapaksha@gmail.com

${ }^{2}$ Department of Building Economics, University of Moratuwa, Sri Lanka, psridarran@uom.lk

${ }^{3}$ Department of Building Economics, University of Moratuwa, Sri Lanka, dulinirathnayake@gmail.com
} 
considerable studies conducted to explore application of AR in the field of facilities management and building maintenance except for few that are related to construction field (Rajaratnam et al., 2021), but not specifically addressing the maintenance phase of a building. Therefore, with this research it is intended to explore the applications of AR for efficiency in the maintenance phase of a building in Sri Lanka. Proceeding with this aim, one of the main objectives is to gain a wide perspective on features of AR technology and its application on building maintenance through a literature survey. Followed by this objective, it is also intended to identify the issues that are currently faced by the maintenance technicians in Sri Lanka when conducting their activities how AR can be used as a solution to address and remedy these identified issues with the intention of making the maintenance activities more efficient.

\section{LITERATURE REVIEW}

\subsection{AUGMENTED REALITY}

Many authors adopt Azuma's definition of Augmented Reality which provides a more descriptive and deeper explanation given by identifying 3 major requirements of the technology (Adikari, et al., 2020; Chen, et al., 2020; Katiyar, et al., 2015).

- Combines real and virtual objects in the real environment

- Interactive in real time and

- Registered in 3 Dimensions

Augmented Reality enables simultaneous interactions between virtual objects such as text, images, 3D models, music, and video (Chen et al., 2020) and real objects in the real time, so that any problem or issue can be analysed efficiently with the help of these virtual and real objects (Li et al., 2017). This way, the two types of objects blend potently with each other (Chen et al., 2020) enhancing the sense of reality through integration of virtual and real objects in real time (Alkhamisi and Monowar, 2013). Another interpretation for Augmented Reality can be derived from the concept of mixed Reality through introduction of "virtuality continuum" which demonstrates a mixture of classes of objects in a display situation (Milgram et al., 1994). As illustrated in Figure 1, this model discusses two ends; Real environment which is visible to the naked eye without any electronic display and Virtual Environment which fully comprises of virtual objects while the Augmented Reality is considered one that is in the intermediate state between the two ends. Nevertheless, the Augmented Reality interface does not occupy a particular point as tend to move along the continuum as per the extent of virtual cues added in order to enhance the user experience (Billinghurst et al., 2014).

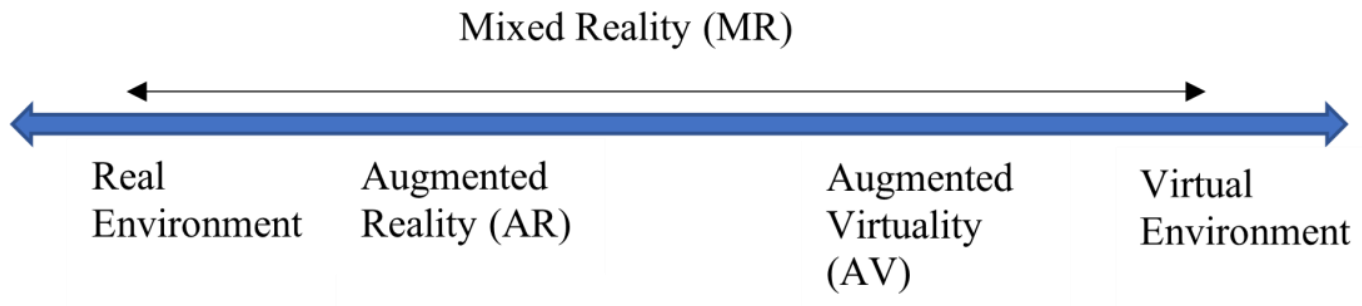

Figure 1: Virtuality continuum (Source: Milgram et al., 1994) 


\subsection{APPlications OF AUgmented REAlity in BUILding Maintenance}

\subsubsection{Information Visualization}

Architecture, engineering, construction and facilities management usually involves a lot of information (Liu and Seipel, 2018), and as facilities managers, it is required to relate the physical objects to this information database (Gheisari et al., 2014). By incorporating Augmented Reality and Information needed for maintenance activities, a framework was introduced after a thorough study of the industrial challenges faced (Amo et al., 2018). It focusses on diagnosis, repair and analysis tasks and defines that information systems and maintenance environments connected to the activities as shown in the figure. Accordingly, it uses the sensor data and creates 3D models helping the technician to correctly diagnose the issue. For repairing, 3D animated Real Environment Augmented Reality (AR) instructions can be given for the repair task identified and furthermore, the captured data in previous processes can be used for further improvement. It is also argued that real time data are essential for making prompt decision and diagnosing problems correctly (Natephra and Motamedi, 2019) and has been remedied using an Internet of Things (IoT) platform with sensors incorporating with Augmented Reality. For instance, an energy data visualization system has been developed which provides the user with energy parameters such as consumption, voltage, current of different appliances (AlonsoRosa et al., 2020). With the help of IoT sensors, cloud computing and Augmented Reality, this system has shown positive result in terms of maintenance personnel's habits and usability perception. Augmented Reality also enables visualization of installations and facilities such as power lines and plumbing that provides better experience for the user (Liu and Seipel, 2018).

\subsubsection{Training and Skill Development}

In Augmented Reality Training, one of the major advantages is its ability to allow the worker to interact with real and virtual objects simultaneously with the required information and guidance in virtual form (Di Cecca et al., 2016; Ferrise et al., 2013; Schwald and Laval, 2003; Webel et al., 2013). It displays information such as technical description on task components, task progress, and critical information (Gavish et al., 2011), instructions and location based information directly over real objects (Webel et $a l ., 2013)$. Overlaying of such virtual information on real environment helps to reduce cascading of errors by eliminating the reliance on erroneous landmarks that can be results of the previous step and has proved to reduce error rate (Tang et al., 2003).In addition the above, technician training by Augmented Reality means has other advantages such as technician's attention on cautions and warnings and improvement of technician's ability to discriminate different operational conditions (Di Cecca et al., 2016). Also, Tang et al. (2003) point out that overlaying of information reduces the time taken for searching manuals, thus boosts the efficiency of the training process.

\section{RESEARCH METHODOLOGY}

A qualitative approach is selected on the premise that the aim of this research is to explore the viability of Augmented Reality for efficient building maintenance in Sri Lanka and it highly involves questions starting with "how" and "why" and it is quite hard to scale it with a numerical value. As the research method, case study stands out as a viable method for this particular research as it is a powerful tool to answer "how" and "why" questions in depth in a holistic manner (Ebneyamini and Moghadam, 2018). According to Yin 
(2003), a case study may comprise of a single case or multiple cases and therefore, based on the phenomenon, the writer should select the most appropriate number of cases for the study (Gustafsson, 2017). On this basis, since making a project proposal presenting a solution for the identified issues using AR was an objective of the major research, selecting a single case seemed more practical. Therefore, a case is seleced on the criterion on whether or not the building has in house maintenance program, the unit of analysis chosen is the issues or causes for delays faced by maintenance technicians in the selected building. Online interviews are used to collect data related to the issues currently faced from maintenance technicians as they are te front liners in maintenance activities of a building and expert interviews with 2 professionals in the field of providing IoT based solutions for building management is later conducted in order to find solutions to these issues with the help of Augmented Reality. The method that is used to analyse data is content analysis.

\section{ANALYSIS AND DISCUSSION}

\subsection{ANALYSIS OF DATA COLLECTED FROM THE INTERVIEWS WITH THE TECHNICIANS}

The collected data is analyzed under 5 main categories in which data was collected and the issues faced by maintenance technicians were identified.

\subsubsection{Issues Related to Comprehension of the Instructions Received}

According to the answers received, it was mentioned that the mean of receiving instructions to perform tasks was in verbal content. Usually, a brief meeting is taken every morning explaining the technicians regarding the activities that are scheduled and instructions are given during this time. Other than that, any other activity that requires immediate attention are communicated to the technicians through a mobile phone call which is also carried out in a verbal manner. Regarding the clarity of the instructions received, half of the respondents had no issues heeding the instructions while the rest faced issues understanding the instructions given by the executives and managers.

Especially, those technicians with less experience and knowledge would often get confused when a task is assigned only with words. It would require extra inputs and effort to convince them the intention of the task and the required steps to be followed in the process. It seems that the instructions in verbal form has a tendency to create difficulties in heeding the gist of what is being said to certain technicians making the tasks inefficient in the long run.

The first issue identified is in regard to technicians comprehending the instructions received by them when tasks are assigned to them. This showed some deviation from the issues highlighted in literature findings. "Less experienced" technicians with experience levels "1-5 years" as well as comparatively experienced technicians with "5-10 years" experience answered that they faced confusions with verbal instructions. The response they gave regarding using animation-based instructions seems to pave the way for integration of augmented reality. 


\subsubsection{Issues Related to Accessing Standard Operating Procedures, Instruction Manuals and Similar Media}

The responses revealed that the technicians are using these documents considerably but, there are no SOP/instruction manuals and other similar media in adequate level. When inquired about the location and accessibility of these documents, a questionable scenario was arisen as $50 \%$ of them responded saying that these documents are not accessible while they were accessible to the rest. It was asked on their preference to use animationbased instructions instead of text-oriented media and all of them extended their preference over this.

It is evident that half of the population of technicians are unaware on the availability of SOP and other similar media that are useful in executing tasks that are considerably alien to them that are out of the routine tasks. The accessibility to such media seems to be an issue prevailing in the facility. Instead of these text-based instructions, the technicians were highly willing to use some type of animated instructions overlaying the work carrying out.

\subsubsection{Issues Related to Building Inspection}

The answers given to the questionnaire showed that all the technicians are involved with the inspection activities of the building and they face issues in accessing the building elements from time to time. It was even mentioned that the building service they face accessibility issues the most is Heating Ventilation and Air Conditioning system while electrical system and elevators and escalators rank the second. In addition to accessibility issues, they also face safety concerns when performing these inspection activities.

The results showed availability of inconveniences when performing building inspections accessing the building elements subjected to inspection. The reason for that could be due to heights, hindrances from other elements or safety concerns. Due to this reason, the technicians are more likely not to perform the tasks up to required standards or quality that can cause greater loses in the long run.

The third issue focusses on the accessibility and safety concerns during inspection activities, which was responded by technicians stating they "sometimes" face issues when inspecting building elements in terms of accessing, safety and various other aspects. According to the responses received, the main building services related to which these issues arise are Heating Ventilation and Air Conditioning system, Electrical system and elevators and escalators.

\subsubsection{Issues Related to Data Retrieval}

The data collected showed that they are making use of history of maintenance tasks related to each asset and the most common way of retrieving these documents was asking from the supervisor while they also refer to the maintenance records by themselves if possible. It was even revealed that sometimes the tasks get delayed because of the unavailability of maintenance records and uncertainties of the previous maintenance activities done to certain assets.

The common method of getting history data which is coordinating with supervisor that seems to take considerable amount of time retrieving the required data that in turn makes the activities inefficient. The technicians were prone to idling that leads to low productivity during this data retrieval process. It also appears to rely on human activity 
and therefore possibility of occurrence of errors is also high. The other method of getting required data is the accessing them by themselves that incur considerable time as they often need to make several trips to find the desired log sheets and other documents that in result causes some delays performing tasks.

The final issue that was prevalent among the technicians of the facility is in relation to retrieval of the maintenance history data. It was the response of all the technicians that they "ask from the supervisor" for any information that they require. It was highlighted by them that "sometimes" their tasks get delayed due to lack of information that are essential for the activity. It is straightforward that their mode of data retrieval involves two parties - the technician and the supervisor and therefore, it is more time consuming and it even raises questions in terms of reliability.

\subsection{ANALYSIS OF DATA COLLECTED FROM THE EXPERT INTERVIEWS}

It was idea of the experts that the mentioned that the facility requires a virtual server comprising of data related to each of the assets, their current conditions and the concept of Building Information Modelling (BIM) that acts as a database of all the asset related as well as maintenance related information that can be easily retrieved comes into play in this regard. In addition to data retrieval, it is also in integration with the IBMS of the building that allows users to control the assets from the 3D view. However, this system is built on a complete virtual platform which could only be accessible for maintenance executives and managers who are privileged with personal computers. Therefore, in order to fit the use case especially to technicians, a separate system involving Augmented Reality should be built by considering the mentioned program as a basis. This would provide solutions to the issue arisen when retrieving maintenance history records and other asset data.

It was further stated that their company currently possess systems that provides instructions to perform certain maintenance tasks but this system only covers preventive maintenance routine tasks that are programmed beforehand. This would satisfy the requirement of the facility in providing instructions to technicians who would not comprehend when communicated verbally, but it would be only applicable to routine tasks. It was suggested to incorporate this system with another system that they are currently developing aiming at corrective workorder generation that involves automatic detection of issues with assets and providing technician with what task is to be carried out. It makes use of an algorithm similar to a root cause analysis and finds the real reason for the failure of the asset. It can be programmed to provide the end user with what action to be taken to fix the problem with animated instructions projected in real time.

\subsection{DiscusSiON OF APPLICABILITY OF AUGMENTED REALITY TO ADDRESS THE IDENTIFIED ISSUES}

Solutions to identified issues have been explored with the help of the literature review as well as with the inputs given by the experts who are engaged in providing IoT based solutions for building management.

A number of cases can be quoted from literature where Augmented Reality based systems have been adopted in order to remedy similar problems mentioned above. For instance, the framework in Figure 2 introduced by Amo et al. (2018) diagnoses breakdowns with the help of sensor data and provides the necessary instructions in $3 \mathrm{D}$ animated fform 
andaddressed more effectively with Augmented Reality. It not only helps technicians in receiving instructions even with the lack of SOP, but also minimizes the time taken to diagnose the issue causing the failure.

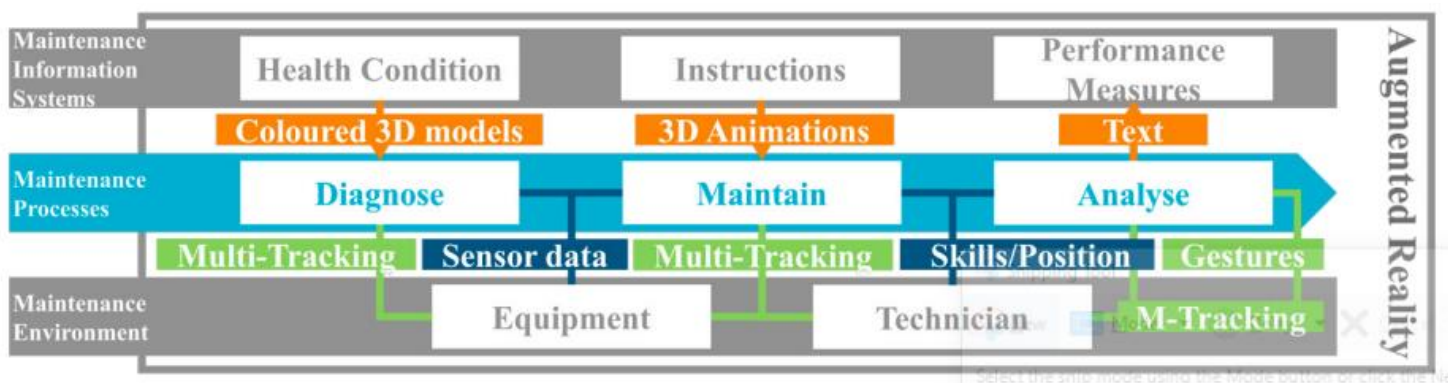

Figure 2: Framework for use of AR technology for maintenance activities (Source: Amo et al., 2018)

A system for "image based indoor localization" incorporating Augmented Reality was developed by Baeka et al. (2019) targeting sanitary pipes, but it is reasonable to assume that a similar model would provide solutions to the problem in this particular case. It makes use of "real time data" collected from sensors which are controlled with a "Building Information Model (BIM)" as the base.

From the expert interview, it is revealed that a "virtual server" is needed to act as the database and the concept of "Building Information Modelling (BIM)" is important in that regard. Accordingly, it would act as the base for the whole system while integrated with Augmented Reality to facilitate visualization of required "information and instructions" for end users. Data is made abundant and access to them is made instant with such a reliable database.

In addition, it is the opinion of the experts that integration of "automatic workorder generation" program with this system in order to include corrective maintenance instructions in addition to the prior arranged preventive maintenance routine tasks. This system would run an algorithm similar to a "root cause analysis" and conclude on what element is causing the failure and propose corrective actions and Augmented Reality is used to visualize the steps included in the actions in real time.

The proposed system would mainly comprise of two major features.

1. Visualization of information - Addressing the issues discussed in sections 4.1.2, 4.1.3 and 4.1.4

- Maintenance records, asset data and other related data

- Real time data

2. Visualization of instructions - Addressing the issues discussed in sections 4.1.1 and 4.1.2

- Visualization of instructions for routine preventive maintenance tasks

- Visualization of instructions for corrective maintenance tasks

\subsubsection{Architecture of the Working Principle of the Proposed System}

As depicted in Figure 3, Building Information Model acts as the server or the repository encompassing the required information while processing the incoming inputs. Inputs are fed to the BIM through the IoT sensors providing real time information while other asset data and maintenance records can be inserted to the server manually. It also receives 
signals coming from the system that automatically detects corrective actions required for breakdowns which functions separated from this program.

When the camera of the device is directed towards a certain asset, it tracks the markers that are attached to the asset and request is sent to the BIM. This request could be either or a combination of request for maintenance history, active cases, PM tasks steps or real time information. This request is processed in the BIM server and then relevant information is fed to the virtual layer with the positions of the markers so that the virtual elements such as texts, symbols, charts, graphs, animations could be overlaid in the real time at correct geographical location. When the task is completed, a report is automatically given to the BIM that stores the information for future use. The below Figure 3 summarizes the basic Architecture of the proposed Augmented Reality based system that aims at solving the identified issues of the selected building.

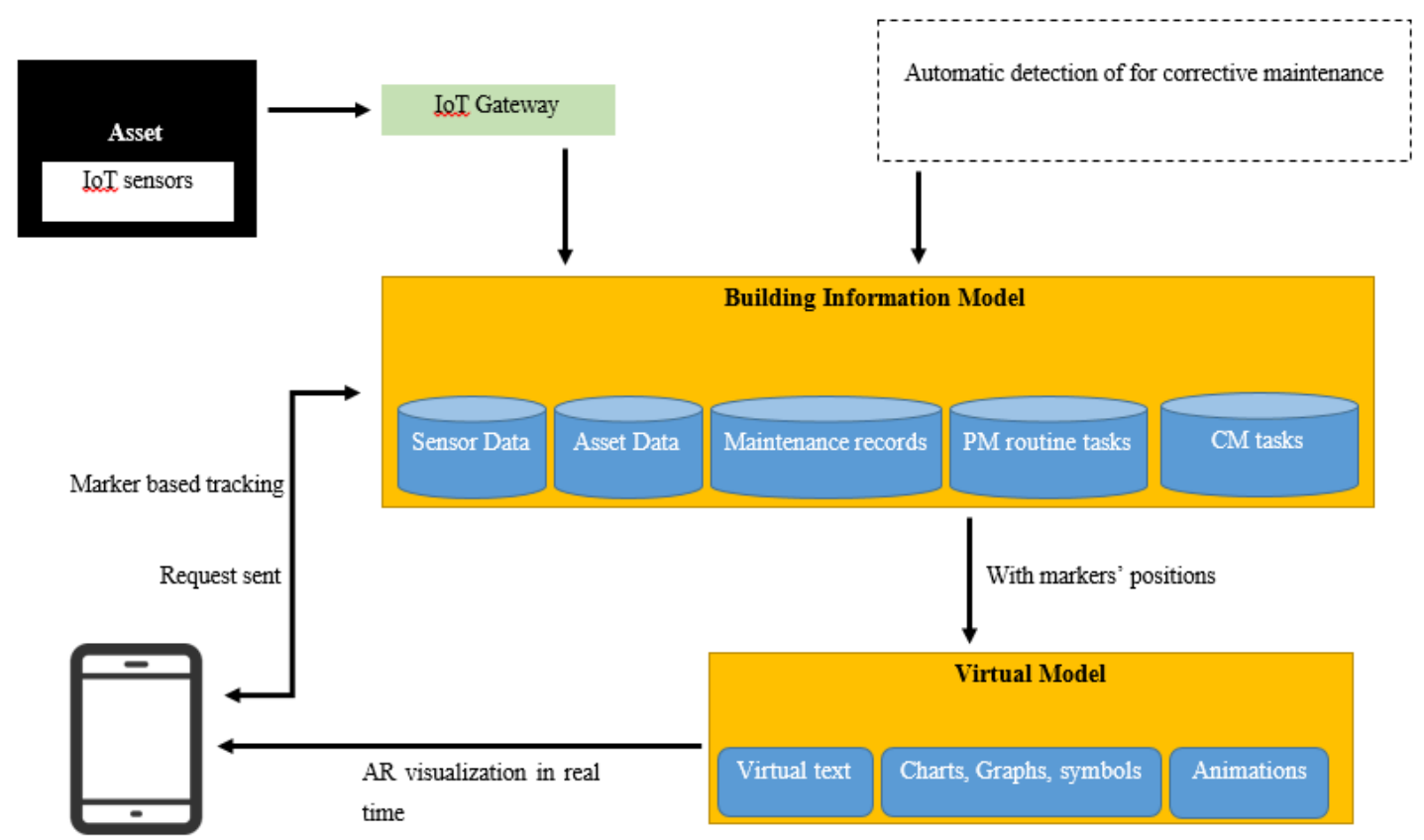

Figure 3: Architecture of the proposed Augmented Reality based maintenance system

\subsubsection{Other Advantages of using an Augmented Reality based System for the Facility}

It is straightforward that this system basically remedies the identified issues of the facility, but it is also evident from the literature survey that it carries other advantages as well.

1. Reduction of time spent on head and neck movement during task

A study conducted by Henrysson et al. (2005) emphasizes on the fact that "there is no need for distracting domain switching", as the user can concentrate solely on the task being carried out without distracting between paper-based manual and the equipment. In this model, augmented reality enables this benefit to the user providing an exclusive experience integrated with real time interaction.

2. Facilitates informed decision

From the interview with the technicians, the respondents revealed that their activities "get delayed due to lack of information or time taken to receive the information". It is clear 
that the decisions they make often do not take information into consideration due to this situation. But with this system, the maintenance related decisions they take would be more reliable, information based and with least amount of error.

3. Allows employment of less skilled technicians

With the instructions in the animated form, even technicians with less experience can conveniently carry out the tasks (Fiorentino et al., 2014) thus leading to cost savings on high skilled technicians, time savings and less human error.

4. Training and skill development

Although training is not a primary purpose of the proposed system, the animation based instruction visualization provides a sound platform for novice technicians to carry out tasks and many augmented reality based systems have shown success in training and skill development of the technicians (Damiani et al., 2018). Similarly, in this model, the visualization of instructions for preventive as well as corrective tasks provides an opportunity for user to learn and develop his skills.

\section{Virtual repository of all information}

As discussed, the architecture of the system shows that Building Information Model acts as the central server storing information such as maintenance records, real time data, and instructions. It is clear that it eliminates the need for hard bound copies which had to be accessed through "calling supervisors" when technicians needed to refer them. A more reliable repository of information is created as a result of implementation of this system in addition to making maintenance activities efficient.

\subsubsection{Possible Barriers for the Proposed AR based System}

1. Developing a BIM for an existing building

The expert interview revealed that a Building Information Model is usually built for a "proposed building" with abundant of information to be incorporated with the model, but developing one for an existing building could be "challenging" and "complex" resulting high costs for the client. There could be possibility of required data being unavailable or not adequate to build the model that would result an unsuccessful end product.

\section{Adopting to a new system}

The facility not implementing any system that is similar to the proposed system, it is reasonable to expect a resistance or a reluctance from technicians. Therefore, it would require a separate workshop or a training program to introduce and train them on the applications and benefits of the proposed system. However, from the response received showing their preference to "animation-based instructions", a positive reaction and embracing of the new technology can also be expected from the technicians. Nevertheless, making the users adopt to a new system could be challenging, therefore suitable strategies will have to be exercised by the management of the facility.

\section{CONCLUSIONS AND RECOMMENDATIONS}

AR which is a novel concept that is applicable in numerous fields is adopted in the field of Facilities Management especially in relation to building maintenance in order to make the processes efficient. The major aim of this study which was to establish the application of AR in making the maintenance tasks in a building more efficient was first achieved by 
gaining a thorough knowledge on applications of this concept worldwide in building maintenance as well as other fields by conducting a literature survey. Proceeding to the second objective which was to identify the issues faced by maintenance technicians in Sri Lanka that causes delays and inefficiencies, interviews were held with them that revealed 4 major issues that were related to mode of instructions given, inconvenient access to SOP and similar media, issues when performing inspection activities in terms of safety and accessibility and retrieval of history data and asset data. Following up with these issues, the final objective which was to remedy them using the concepts of AR was achieved with the inputs of the experts in the field of providing IoT based solutions for building management as well as the knowledge gathered from studying the previous work carried out by other researchers globally in the literature review. With the use of a Building Information Model and Augmented Reality, a system is proposed to address these issues and it has the potential of leading to cost and time savings, high customer satisfaction that ultimately results in high profitability. However, there could be challenges in developing a Building Information Modell to a prevailing building and also in making the technicians adopt into a new system. Through this research, it is recommended to conduct more comprehensive research to identify causes and issues faced by frontline maintenance technicians making the maintenance practices inefficient and explore the applications of novel concepts such as Building Information Modelling, Internet of Things and Augmented Reality in building maintenance for Sri Lanka.

\section{REFERENCES}

Baeka, F., Ha, I. and Kim, H., 2019. Augmented reality system for facility management using image-based indoor localization. Automation in Construction, 99, pp. 18-26.

Adikari, S.B., Ganegoda, N.C., Meegama, R.G. and Wanniarachchi, I.L., 2020. Applicability of a single depth sensor in real-time 3D clothes simulation: Augmented reality virtual dressing room using kinect sensor. Advances in Human-Computer Interaction, 2020, pp. 1-10.

Alkhamisi, A. and Monowar, M., 2013. Rise of augmented reality: Current and future application areas. International Journal of Interest and Distributed Systems, 1(4), pp. 25-34.

Alonso-Rosa, M., Gil-de-Castro, A., Moreno-Munoz, A., Garrido-Zafra, J., Gutierrez-Ballesteros, E. and Cañete-Carmona, E., 2020. An IoT based mobile augmented reality application for energy visualization in buildings environments. MDPI Applied Sciences.10(2), pp. 600-605

Amo, I.F.D., Erkoyuncu, J.A., Roy, R. and Wilding, S., 2018. Augmented reality in maintenance: An information-centered design framework. Bremen, 19, pp. 148-155.

Billinghurst, M., Clark, A. and Lee, G., 2014. A survey of augmented reality. Foundations and Trends in Human Computer Interaction.6(4), pp. 355-385

Chandike, B.T., 2016. Study on applying "Augmented Reality" for effective learning of school curriculum of advanced level in Sri Lanka. International Journal of Scientific and Technology Research, 5(10), pp. 242-246.

Chandrasekara, T., 2015. Rejuvenating dysfunctional public spaces using Augmented Reality Systems (ARS). American Journal of Mobile Systems, Applications and Services, 1(1), pp. 64-76.

Chatzopoulos, D., Bermejo, C., Huang, Z. and Hui, P., 2017. Mobile augmented reality survey: From where we are to where we go. IEEE ACCESS, 5, pp. 6917-6950.

Chen, Y.J., Lai, Y.S. and Lin, Y.H., 2020. BIM-based augmented reality inspection and maintenance of fire safety equipement. Automation in Construction.110, pp. 30-41

Damiani, L. Demartini, M., Guizzi, G., Revetria, R. and Tonelli, F., 2018. Augmented and virtual reality applications in industrial systems: A qualitative review towards the industry 4.0 era. IFACPapersOnLine 51(11), pp. 624-630.

Di Cecca, C. Ciuffini, A. F., Ferrise, F., Mapelli, C. and Gruttadauria, A., 2016. Study about the augmented reality adoption in the maintenance in steelmaking area. La Metallurgia Italiana, 2016(7), pp. 11-16. 
Ebneyamini, S. and Moghadam, M.R.S., 2018. Toward developing a framework for conducting case study research. International Journal of Qualitative Methods, 2018, pp. 1-11.

Ferrise, F., Caruso, G. and Bordegoni, M., 2013. Multimodal training and tele-assistance systems for the maintenance of industrial products. Virtual and Physical Prototyping, 8(2), pp. 113-126.

Fiorentino, M., Uva, A., Gattullo, M. and Debernardis, S., 2014. Augmented reality on large screen for interactive maintenance instructions. Computers in Industry, 65(2), pp. 270-278.

Gavish, N., Seco, G., Webel, S. and Rodriguez, J., 2011. Transfer of skills evaluation for assembly and maintenance training. BIO Web of Conferences, 1(2011), pp. 1-4

Gheisari, M., Williams, G., Walker, B. N. and Irizarry, J., 2014. Locating building components in a facility using augmented reality vs. paper-based methods: A user-centered experimental comparison. Computing in Civil And Building Engineering,1(2014). pp. 850-857.

Gustafsson, J., 2017. Single case studies vs multiple case studies: A comparative study. Halmstad, Sweden: Halmstad University.

Henrysson, A., Ollila, M. and Billinghurst, M., 2005. Mobile phone based AR scene assembly. Mobile and Ubiquitous Multimedia, 1(2005), pp. 95-102

Katiyar, A., Kalra, K. and Garg, C., 2015. Marker based augmented reality. Advances in Computer Science and Information Technology (ACSIT), 2, pp. 441-445.

Liu, F. and Seipel, S., 2018. Precision study on augmented reality-based visual guidance for facility management tasks. Automation in Construction, 90, pp. 79-90.

Li, W., Nee, A.Y. and Ong, S.K., 2017. A state-of-the-art review of augmented reality in engineering anaysis and simulation. Multimodal Technologies and Interaction, 1(3), pp. 1-17

Milgram, P., Takemura, H., Utsumi, A. and Kishino, F., 1994. Augmented reality: A class of displays on the reality-virtuality continuum,.77(12), pp. 282-292.

Natephra, W. and Motamedi, A., 2019. Live data visualization of IoT sensors using Augmented Reality (AR) and BIM. In: Proceedings of the 36 ${ }^{\text {th }}$ ISARC, Banff Alberta, Canada 21-24 May 2019, Alberta Canada: University of Alberta, pp. 632-638.

Rajaratnam, D. Weerasinghe, D.M.L.P., Abeynayake, M., Perera, B.A.K.S. and Ochoa, J.J., 2021. Potential use of augmented reality in pre-contract design communication in construction projects. Intelligent Buildings International.1(2021), pp.1-24.

Schwald, B. and Laval, B., 2003. An augmented reality system for training and assistance to maintenance in the industrial context. Journal of WSCG.11(6), pp. 1-8.

Tang, A., Owen, C., Biocca, F. and Mou, W., 2003. Comparative effectiveness of augmented reality in object assembly. Paper: New Techniques for Presenting Instructions and Transcripts, 1(2013), pp. 7380 .

Wang, X., Kim, M. J., Love, P. and Kang, S.-C., 2013. Augmented reality in built environment: Classification and implications for future research. Automation in Construction, 32, pp. 1-13.

Webel, S., Bockholt, U., Engelke, T., Gavish, N.,Olbrich, M. and Preuschec, C., 2013. An augmented reality platform for assembly and maintenance skills. Robotics and Autonomous Systems, 61(4), pp. 398-403.

Yin, R., 2003. Case study research: Design and methods. Thousand Oaks, CA: Sage. 